\title{
Autonomous thermal camera system for monitoring the active lava lake at Erebus volcano, Antarctica
}

\author{
N. Peters ${ }^{1}$, C. Oppenheimer ${ }^{1}$, and P. Kyle ${ }^{2}$ \\ ${ }^{1}$ Department of Geography, University of Cambridge, Downing Place, Cambridge, CB2 3EN, UK \\ ${ }^{2}$ New Mexico Institute of Mining and Technology, Socorro, USA
}

Correspondence to: N. Peters (njp39@ cam.ac.uk) and C. Oppenheimer (co200@ cam.ac.uk)

Received: 3 September 2013 - Published in Geosci. Instrum. Method. Data Syst. Discuss.: 25 October 2013

Revised: 20 January 2014 - Accepted: 29 January 2014 - Published: 5 February 2014

\begin{abstract}
In December 2012, the Mount Erebus Volcano Observatory installed a thermal infrared camera system to monitor the volcano's active lava lake. The new system is designed to be autonomous, and capable of capturing images of the lava lake continuously throughout the year. This represents a significant improvement over previous systems which required the frequent attention of observatory researchers and could therefore only be operated during a few weeks of the annual field campaigns. The extreme environmental conditions at the summit of Erebus pose significant challenges for continuous monitoring equipment, and a custom-made system was the only viable solution. Here we describe the hardware and software of the new system in detail and report on a publicly available online repository where data will be archived. Aspects of the technical solutions we had to find in order to overcome the challenges of automating this equipment may be relevant in other environmental science domains where remote instrument operation is involved.
\end{abstract}

\section{Introduction}

Situated on Ross Island, Antarctica, the 3794 m-high crater of Erebus volcano has played host to an active phonolite lava lake since at least 1972 (Giggenbach et al., 1973). Persistent lava lakes are a spectacular but rare form of open vent volcanism exhibited by just a handful of volcanoes around the world. As the exposed top of the volcano's plumbing system, they provide a particularly valuable opportunity to observe directly the magmatic processes that are normally hidden from view. The comparatively benign nature of most of these lakes' activities allows ground-based measurements to be made in relative safety. Much has been learned about magmatic processes simply by studying time-lapse photographs and video recordings of lava lakes (for example Orr and Rea, 2012; Dibble et al., 2008).

Thermal infrared (IR) cameras are an important tool in the study of lava lakes and have been used extensively in a number of lake studies around the world, ranging from quantification of radiative heat output (e.g. Calkins et al., 2008; Oppenheimer et al., 2004) to studying surface velocity (Oppenheimer et al., 2009). They are also used more widely as an operational tool in volcano monitoring (e.g. Spampinato et al., 2011). Although absorption of infrared radiation by volcanic gases makes accurate temperature measurements difficult to achieve (Sawyer and Burton, 2006), the ability to image through optically opaque volcanic plumes gives IR cameras a significant advantage over conventional cameras for monitoring lava lakes.

The Mount Erebus Volcano Observatory (MEVO) has been operating a thermal camera on Erebus during annual field campaigns (typically from late November to early January) since 2004. However, due to the many challenges of working in such an extreme environment, the time series of IR images tend to be very short and fragmented. In 2010 the decision was made to upgrade the thermal camera to a system that could potentially (contingent on a reliable power supply) run autonomously and continuously year-round. After two years of development and testing, the new system was installed during the 2012 field season.

Autonomous instruments have become a popular choice for researchers from a variety of disciplines working in Antarctica. Although it might seem an obvious solution to obtaining long time series measurements without the need 
Table 1. Approximate cost breakdown of the thermal camera system.

\begin{tabular}{lr}
\hline Component & Approximate Cost \\
\hline Thermal Camera & $£ 20000$ \\
Single Board Computer & $£ 300$ \\
GPS & $£ 80$ \\
Solid-state Hard Disc & $£ 300$ \\
Sensors, SSR and Interface & $£ 200$ \\
Enclosure (inc. IR window) & $£ 400$ \\
\hline
\end{tabular}

for extended field campaigns, the technical challenges of creating such systems are numerous (see for example Bauguitte et al., 2011; Lawrence et al., 2004). MEVO already operates several year-round seismic stations (Aster et al., 2004); however, the location of the thermal camera system and its high data rate meant that it presented many new challenges.

The aims of this article are threefold: (i) to describe the hardware and software of MEVO's new thermal camera system in detail in the hope that the experience we gained through its development may be beneficial to others hoping to undertake similar projects; (ii) to document the design and capabilities of the control software developed for the project and to make it freely available as a useful starting point for other camera projects; and (iii) to publicise the newly created data archive for the thermal camera images from Erebus volcano, which may be freely accessed by those interested in lava lakes, magma rheology etc.

\section{Hardware}

The camera system is mostly an aggregation of off-the-shelf parts, with very little custom-made hardware involved. Although this has meant that some compromises have had to be made in terms of specifications, the reduction in cost and development time more than outweighs the disadvantages. Figures 1 and 2 show the main components used, which are discussed in detail in the following sections. The approximate costs of the components are outlined in Table 1. It should be noted however that the control system could be used with any (possibly cheaper) camera unit, provided that it supports the GenICam interface.

\subsection{Camera}

The thermal camera unit itself is a FLIR SC645, with an uncooled $640 \times 480$ element detector, spectral range of $7.5-13 \mu \mathrm{m}$, and a spatial resolution of $0.69 \mathrm{mrad}$. This unit was chosen because, at the time of purchase, it was one of the few thermal cameras that allowed digital output of images in real-time (rather than storing them on an on-board storage medium such as a compact flash card). Furthermore, it supports the non-proprietary GenICam interface, which al-

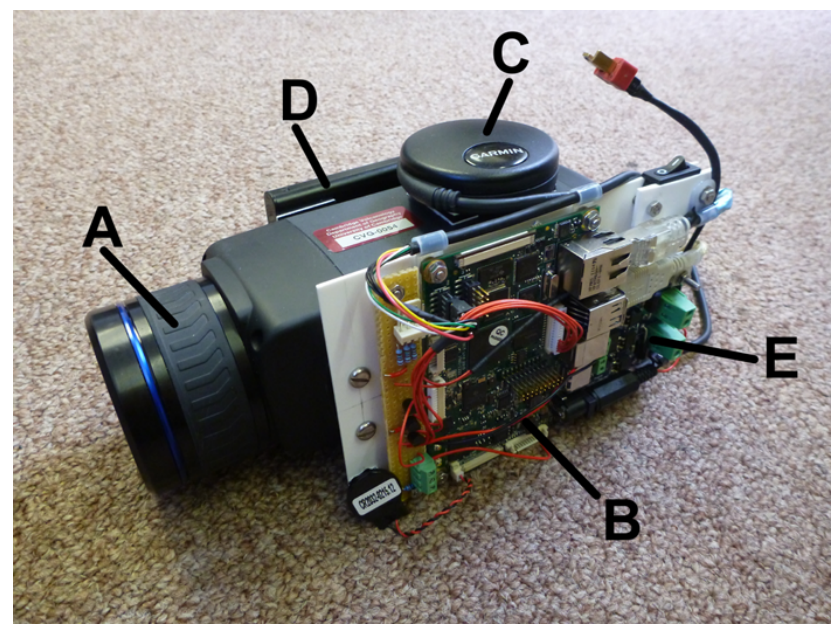

Fig. 1. The main components of the camera system: (A) FLIR SC645 IR-camera, (B) BCT-RE2 SBC, (C) Garmin GPS 18 LVC, (D) Intel 256 Gb SSD, (E) Phidgets SSR.

lows capture parameters to be set on the camera and images to be received via Ethernet.

The camera has three possible temperature ranges that it may be set to. In general we use the range 273-923 K; however, we have occasionally used the higher temperature range of $573-2273 \mathrm{~K}$. The temperature range used for each image is stored in the PNG header data of the image file.

The camera has a built-in calibration source and surface temperatures could, in principle, be obtained per pixel. However, this would require parameterisation of in-band emissivity of the lava lake surface and atmospheric transmittance. While a reasonable estimate might be made for the former, the atmospheric transmittance is highly variable in space and time due to the fluctuating column amounts of volcanic gases in the optical path between the camera and the lake surface ( $\sim 350 \mathrm{~m}$ range). Several magmatic gas species emitted from the lake absorb in the detector's waveband. In an instantaneous image, the transmittance will likely differ widely for each pixel due to the inhomogeneity of the volcanic plume, and from frame to frame, the plume structure may vary considerably. Thus, no unique value of transmittance would be appropriate to correct all the images to surface temperatures. Sawyer and Burton (2006) has shown how use of open-path Fourier transform spectroscopy to measure absorbing species in the optical path can provide some traction on this problem, but only an imaging system would be able to constrain both the spatial and temporal variability in transmittance (and it would require a vast amount of data processing). Consequently, we have recorded images in the "radiometric" setting on the camera - in which the recorded signal is linearly proportional to in-band radiance reaching the detector. While these data are not well suited to estimating surface temperatures on the lake or radiative power outputs, they are valuable for studying lava lake motion, as discussed in Sect. 4.3. 


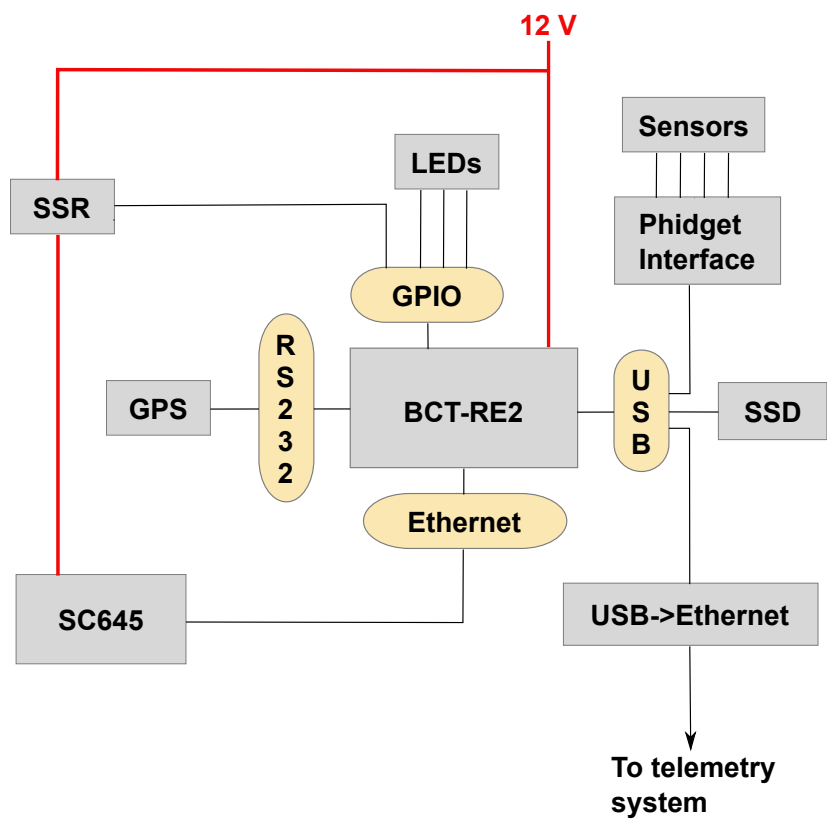

Fig. 2. Block diagram showing the main components of the camera system.

\subsection{Control system}

The control system comprises an ARM-based single board computer (SBC) which is connected to the camera via Ethernet. We used a Blue Chip Technology (BCT) RE2 SBC. Although there is a huge range of SBCs on the market that cover most combinations of interfaces, performance and power consumption, the choice of boards with an extended operating temperature range is considerably more limited. Due to the high computational expense of image compression, we found in testing that we needed a processor of at least $500 \mathrm{MHz}$. We also required multiple USB host ports and several GPIO (general purpose input/output) pins. With its $600 \mathrm{MHz}$ ARM OMAP Cortex A8 processor, $-40^{\circ} \mathrm{C}$ operating temperature and large range of interfaces, the BCT-RE2 met all our requirements at a power consumption of $2 \mathrm{~W}$. The system runs Ubuntu Linux (version 10.10). Additional kernel modules had to be compiled to enable user-space access to the GPIO pins and also to facilitate use of a USB to Ethernet converter.

\subsection{Power supply}

A combination of extreme winds and corrosive gases makes the crater rim of Erebus an unsuitable site for solar panels and wind generators. Instead, power is generated $0.5 \mathrm{~km}$ downslope at the Nausea Knob (NKB) seismic station site, where a $1000 \mathrm{Ah}$ battery bank is charged using a $\sim 0.5 \mathrm{~kW}$ array of photovoltaic panels, and a $100 \mathrm{~W}$ wind turbine. A Schaefer AEP-1500 inverter (chosen for its extended temperature range) is then used to transmit $230 \mathrm{~V}$ through a heavy-duty cable to the crater rim, where it is converted back to $12 \mathrm{~V}$ DC using an REL-185-1004 power supply (also chosen for its extended temperature range).

Based on past experience of unfamiliar researchers causing damage to equipment by connecting it to the wrong power supply, or with reversed polarity, the camera system incorporates a custom-made protection circuit. Reverse polarity protection is provided by a MOSFET, and over-voltage protection is provided by a "crowbar" circuit, which shortcircuits a resettable fuse (PTC) at supply voltages above $15 \mathrm{~V}$. It is our belief that all electronic field equipment should be protected in a similar way. Protection circuits are straightforward to make, cost almost nothing, and prevent simple mistakes from totally destroying equipment. We cannot stress this point enough!

Power to the camera itself is controlled via one of the SBC's GPIO pins using a Phidgets 3053 solid-state relay (SSR). This allows the control software to "hard-restart" the camera in case of problems.

Overall, the system requires $\sim 11 \mathrm{~W}$ of power. Between 6 and $7 \mathrm{~W}$ is used by the camera itself, $\sim 2 \mathrm{~W}$ by the SBC and the remainder by the hard disc.

\subsection{Time synchronisation}

The majority of volcanological research requires the intercomparison of data from many different instruments, making accurate time stamping of the utmost importance. To ensure that the system time on the SBC did not drift, we linked it via its RS232 serial port to a Garmin GPS 18 LVC receiver. The system time was then synchronised with the GPS using standard Linux utilities. Initially we tried the gpsd program; however, we found it to consume excessive system resources and so we switched to the built-in GPS support in ntpd. We had hoped to use the LinuxPPS kernel module to provide very accurate time synchronisation using the PPS (pulse per second) signal output from the GPS. Unfortunately, the RS232 port on the BCT-RE2 is implemented over the I2C bus, and the driver for this does not currently support LinuxPPS. Despite this, we found that synchronisation was possible to within a few milliseconds just by using the NMEA data from the GPS. The control software for the camera has been designed to allow a sufficient delay, following a reboot, for GPS time synchronisation to occur before image capture resumes.

\subsection{Sensors}

The power consumption of the camera system, together with the temperature inside and outside of its enclosure, are monitored using Phidgets sensors. These are connected to the SBC via USB using a Phidgets 1018_2 interface board. Phidgets sensors were chosen for their ease of use and simple software interface.

The data collected by the sensors between December 2012 and April 2013 show a stable power consumption of $\sim 11 \mathrm{~W}$ 


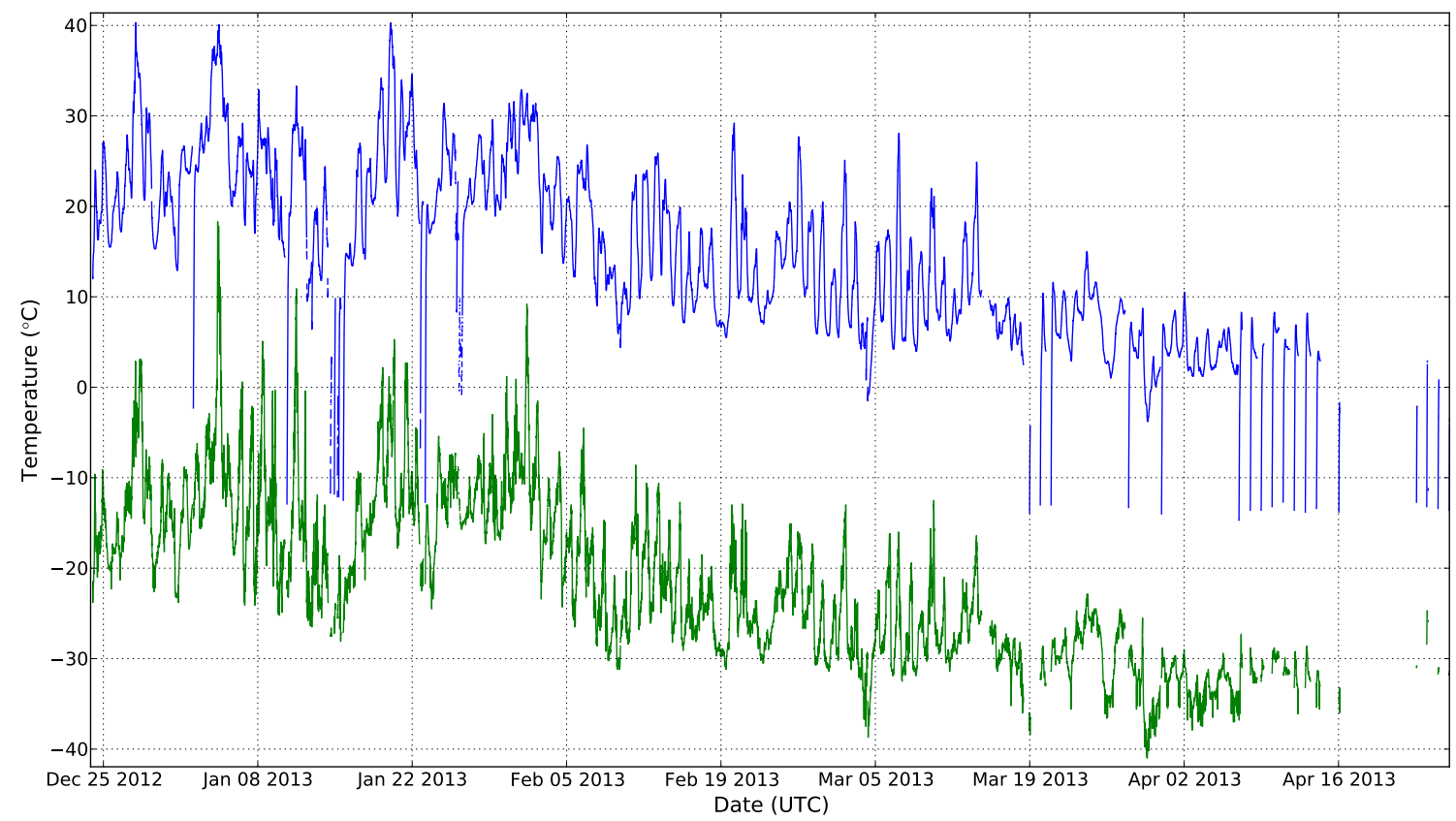

Fig. 3. Time series of temperature readings both inside (upper series) and outside (lower series) of the camera enclosure. It should be noted that the outside temperatures reported appear to be $\sim 10^{\circ} \mathrm{C}$ higher than the actual air temperature.

throughout. Figure 3 shows the recorded temperatures. The sharp drops in internal temperature are caused by power outages, and the subsequent rapid heating clearly demonstrates the ability of the system to keep itself warm simply by using waste heat from the SBC and hard disc. It should be noted that the external temperature sensor seems to over-read by $\sim 10^{\circ} \mathrm{C}$ compared with measurements made with a handheld thermocouple. This is likely caused by heat leakage from the camera enclosure into the external temperature sensor enclosure.

\subsection{Data storage}

The low temperature performance of hard disc drives (HDDs) seems to be poorly researched and/or documented. When choosing a data storage solution for the camera system we were unable to find any high-capacity discs with an extended temperature range. In the end we chose a $250 \mathrm{~Gb}$ Intel solid state drive (SSD). While it was not rated for low operating temperatures, it did have a lower power consumption than conventional HDDs. Some consideration was given to the idea of storing batches of images on the SBC's internal flash memory and then periodically transferring them to the SSD. Although this would have reduced the power consumption of the system, since the SSD would only need to be powered up occasionally, we decided that the risk of data loss due to the increased complexity outweighed the potential benefits.

\subsection{Telemetry}

During the annual MEVO field campaign, a Trango TL-45 $5 \mathrm{GHz}$ wireless Ethernet link is used to telemeter data from instruments at the crater rim back to servers at our field camp. Instruments are connected to the TL-45 via a low-power, low-temperature Parvus PRV-1059 Ethernet switch. The high power consumption of the telemetry system $(\sim 12 \mathrm{~W})$ means that it is only operated during the field campaign (when there is ample solar charging). The thermal camera system incorporates a USB to Ethernet converter to provide it with a second Ethernet interface and allow connection to the telemetry system during this period. Images can then be streamed to the field camp in addition to being stored on the SSD, providing some redundancy in data storage and the ability to monitor the lava lake in real-time.

\subsection{Enclosure}

Previous thermal cameras operated by MEVO were simply wrapped in bubble-wrap to protect them from the cold. Somewhat surprisingly this proved to be very effective. However, the corrosive gases emitted from the volcano rapidly took their toll, and metal parts (including electrical contacts) soon degraded and became unusable. An additional problem with previous systems was that standard tripod mounts were not rigid enough to prevent considerable camera shake caused by the high winds which frequent the summit of Erebus. An air-tight plastic Peli ${ }^{\mathrm{TM}}$ case was used as the enclosure for the new system, to which a rigid aluminium frame 


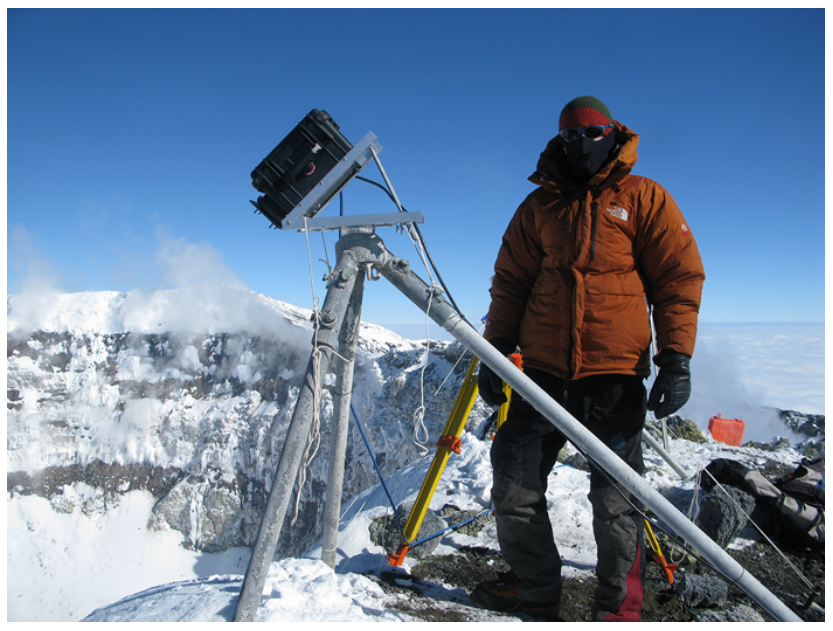

Fig. 4. The new thermal camera system installed at the crater rim of Erebus volcano.

was bolted. The frame was attached to a tripod comprised of scaffolding bars to form a solid mounting which does not vibrate in the wind (Fig. 4). The IR window used in the camera enclosure is $3 \mathrm{~mm}$-thick Germanium with an $8-12 \mu \mathrm{m}$ antireflective coating.

All external fixtures are either aluminium or stainless steel to reduce corrosion. The camera casing has been insulated with expanded polystyrene and all internal fixings are made of plastic to reduce conductive heat loss. Combined with the heat generated by the enclosed electronics, this meant that the temperature inside the enclosure remained above $-5^{\circ} \mathrm{C}$ throughout its operation in 2013 (except during power outages), despite external temperatures of less than $-50{ }^{\circ} \mathrm{C}$ (Fig. 3).

\section{Software}

\subsection{Overview}

The control software for the camera system is comprised of several small programs which are managed by an initialisation script. All programs share a single configuration file, which can be edited to change the operational parameters of the system. The main capture program is responsible for setting the capture parameters of the camera, and then entering an infinite loop of capturing images, performing some basic pre-processing on them and then compressing them as PNG files. During the field campaigns when the telemetry system is in place, two separate server programs are used to send images and sensor data to a viewer program which runs on a computer at the field camp. This allows real-time monitoring of the lava lake and of the thermal camera system itself.

\subsection{Implementation and architecture}

The initialisation script is written in Bash. It is launched automatically on system boot by upstart (a replacement for the System V init daemon) once the networking and GPIO services have been started. The script performs a series of system checks such as ensuring that the SSD is mounted correctly, initialises GPS time synchronisation and then starts the capture and server programs. If errors are encountered that would prevent the capture of images, then the whole system is restarted. An error handling safety net is provided by the kernel watchdog module, which will restart the system if the capture software crashes for some reason or if the system "freezes" (runs out of memory, or has excessive CPU usage). As a final layer of fault tolerance, a crontab entry is used to restart the whole system once a week.

The main capture program is written in $\mathrm{C}++$ and uses libconfig++ for reading the configuration file, libpng for compressing and storing the images, and libaravis (Pacaud, 2011) for interfacing with the camera. The images output from the camera have a bit depth of 16 . To reduce the size of the PNG files produced, they are scaled to a bit depth of 8 before being compressed. In order to preserve as much dynamic range in the pixels representing the lava lake as possible, the images are first thresholded to set all low valued pixels to zero. They are then linearly scaled to 8 bit values as follows:

$x_{8}=\left(x_{16}-\min _{16}\right) \times \frac{255}{\max _{16}-\min _{16}}$

where $x_{8}$ is the 8 bit pixel value, $x_{16}$ is the 16 bit pixel value and $\min _{16}$ and $\max _{16}$ are the minimum and maximum pixel values in the 16 bit image respectively.

Converting the images to 8 bit using this algorithm is sensitive to dead or hot pixels, since these will skew the minimum and maximum pixel values. This is potentially disastrous, as the dynamic range of pixels within the lake in the resulting 8 bit images could be severely impacted. To prevent this from happening, a simple dead/hot pixel detection algorithm is applied to each image before conversion to 8 bit. This works by comparing each pixel to its neighbours and looking for very strong contrasts in value. In order to implement this algorithm efficiently, it ignores the edge pixels of the image, meaning that every pixel analysed has eight neighbours. Edge pixels are simply set to zero. Image files are written to disc using incremental numbers as file names. This method was chosen as it will not result in images being overwritten in the event of unexpected system restarts (e.g. due to power cuts) or loss of system time.

The server programs are written in C. The server for the sensor data uses libphidgets to interface with the sensors. Values read are recorded in a log file and also passed to any connected clients via a socket. The server program for the images works by using inotify to monitor the output directory for the images from the capture program (which is read from the capture program's configuration file). Each new image 

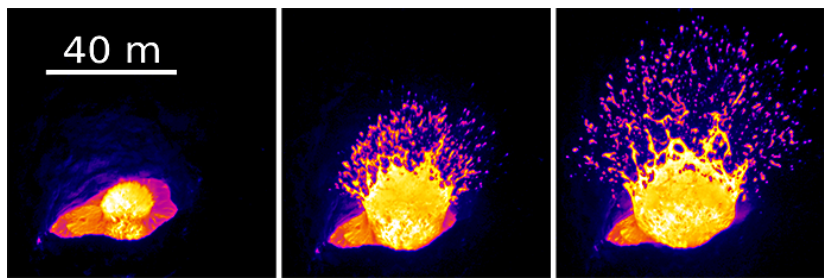

Fig. 5. A sequence of three IR images recorded $\sim 0.17 \mathrm{~s}$ apart, showing the arrival of a large gas slug in the lava lake.

detected is transmitted to any connected clients as a string of raw PNG data, and the user-gpio kernel module is used to flash a status LED connected to one of the GPIO pins of the SBC. The server programs were written as separate programs from the capture program in order to keep the latter as simple, and therefore reliable, as possible.

The viewer program is written in Python and connects to the server programs via a standard socket. Multiple instances of the viewer can connect to the server programs simultaneously.

\subsection{Reuse potential}

All the software created for this project is freely available under the terms of the Gnu Public License from http://dx.doi. org $/ 10.6084 / \mathrm{m} 9$.figshare.784942. Although the image acquisition software is specific to the SC645 camera, fairly minor modifications would enable it to work with any camera that supports the GenICam interface. It is not however suitable for applications that need capture rates above a few frames per second. The image server and viewer programs could be reused "as is" for any image capture application that outputs PNG files, provided that the server side system has inotify.

\section{Results and discussion}

\subsection{The camera system}

The system was installed at the crater rim of Erebus in November 2012 and, other than a few minor issues with the camera being unable to focus to infinity when it was first mounted in the enclosure, it operated without any problems up until the end of the 2012 field season. It was then left running with the intention that it continue to acquire images continuously until the following field season. On returning to the volcano in November 2013, we discovered that the battery bank which serves the camera system had failed. Several of the batteries had cracked casings and all registered $\sim 6 \mathrm{~V}$. The exact cause of this failure was never determined; however, we suspect that it was caused by a faulty wind turbine system which allowed the batteries to over-discharge and subsequently freeze. As a result, power was never restored to the camera after the winter, and therefore images

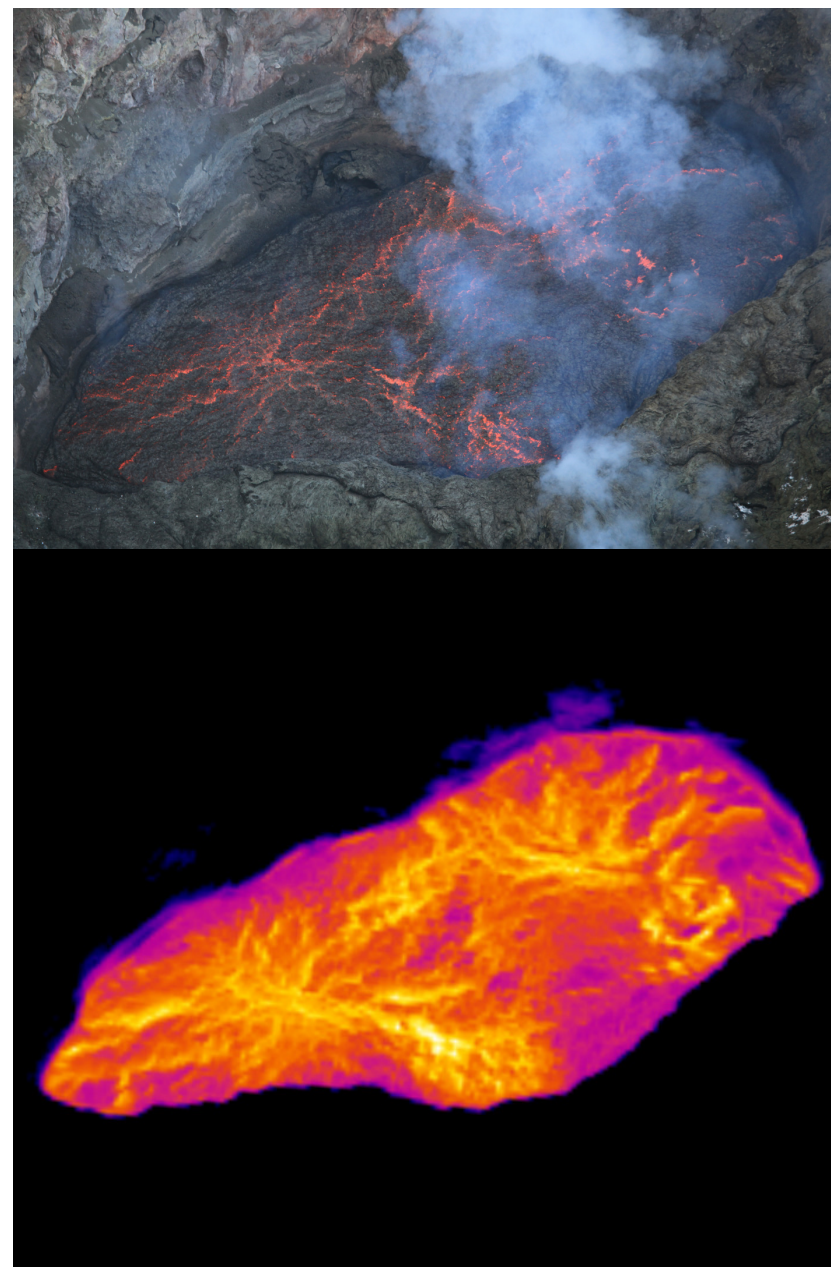

Fig. 6. Simultaneous images from a digital SLR camera and the IR camera. The cracks in the lake's crust are clearly visible in the IR image and can be used to estimate the surface motion of the lake. The ability of the IR camera to image through the volcanic plume is also clearly demonstrated. The lake is $\sim 40 \mathrm{~m}$ across in these images.

were only acquired up until 26 April 2013. It is worth noting however that when power was restored, the system resumed acquisition without any further intervention. The wind turbine and associated controller have now been replaced with a new, redundant system which we hope will provide a greater degree of protection for the battery bank.

A further failure of the system was that the stainless-steel mounting bolt which secures the camera enclosure to the tripod had bent, allowing the enclosure to rotate such that the camera was no longer pointing at the lake. Fortunately, this had happened after the power had failed, and so no additional data were lost. It is somewhat surprising that such a small enclosure can provide enough wind resistance to bend a $5 \mathrm{~mm}$ diameter bolt. We have replaced the bolt with a much larger one, and plans are underway to replace the entire tripod setup 


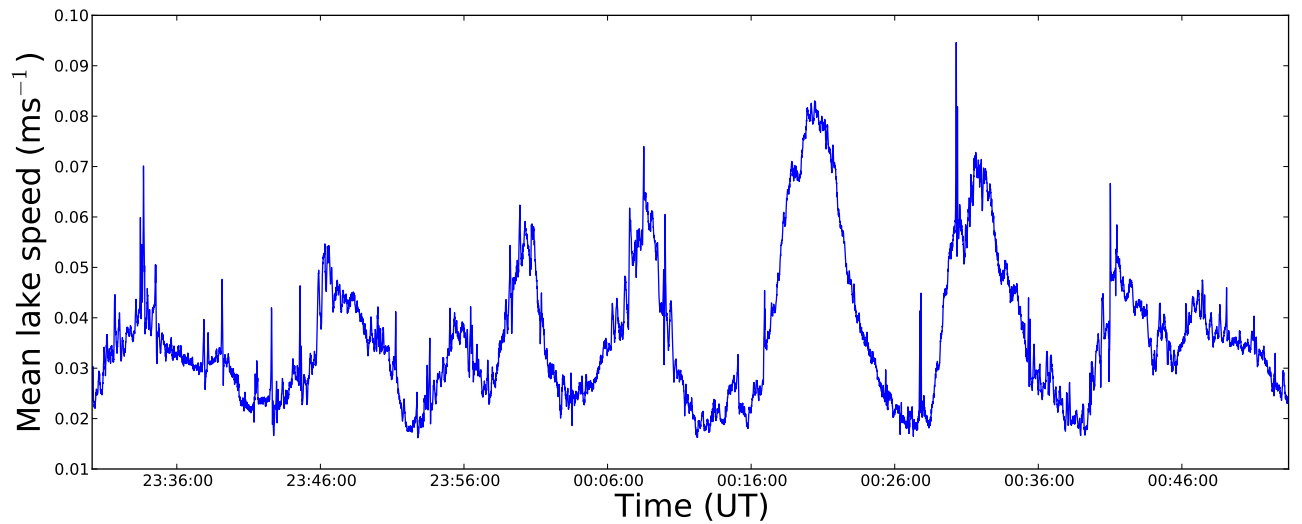

Fig. 7. Time series of mean lake surface speed calculated from IR images captured on 22 December 2010. The pulsatory behaviour of the lake is clearly visible with a period of $\sim 12 \mathrm{~min}$. The sharp spikes in speed ("shot noise") are caused by bubbles reaching the surface of the lake.

with a more substantial mounting system during the 2014 field campaign.

Despite the problems outlined above, the camera system worked well until late April when the sun stopped rising and solar charging ended. Almost four million images were acquired; the largest thermal infrared data set ever recorded on Erebus. The system was left running again after the 2013 field season and, with the improved power system in place, we are hopeful of achieving year-round data acquisition during 2014.

\subsection{The data set}

Figure 5 shows a short sequence of images captured by the camera system during testing in 2010 , which document a large gas slug arriving in the lake. These images were captured at $6 \mathrm{~Hz}$, whereas the camera system ordinarily operates at $0.5 \mathrm{~Hz}$. The data set from the new camera system is freely available at http://www.usap-data.org/entry/ NSF-ANT11-42083/ and the authors would like to encourage researchers with enquiries about its use to contact them. All images in the archive are in PNG format, and contain important meta-data in their file headers. Information about extracting these meta-data can be found in the README file which is part of the archive. At the time of writing the archive contains all the images that were captured by the camera system during the 2012 field season. This will be expanded upon annually as new data are recovered from the camera. Older data sets from previous camera systems will also be made available once the images have been converted into a suitable archive format.

\subsection{Applications}

The Erebus lava lake is covered by a thin crust of cooler material. As the lake convects, this crust cracks and the cracks migrate across the surface driven by the motion of the under- lying lava. Figure 6 shows a comparison between the visible appearance of the lake and an IR image (it should be noted that such a clear view of the lake is exceptionally rare). As can be seen in the figure, the cracks are clearly visible in the IR image and, as demonstrated by Oppenheimer et al. (2009), can be tracked using a wavelet-based motion estimation algorithm (Magarey and Kingsbury, 1998) to determine the surface velocity of the lake. Figure 7 shows a short time series of mean lake surface speed calculated in this way. The pulsatory behaviour of the surface motion is thought to be caused by a density driven bi-directional flow of magma in the conduit feeding the lake (Oppenheimer et al., 2009). A primary goal of the new thermal camera system is to provide long, uninterrupted observations of the pulsatory behaviour of the lake to facilitate a detailed comparison with numerical simulations (Molina et al., 2012) and analogue experiments (Witham et al., 2006; Huppert and Hallworth, 2007) in order to better constrain the magmatic processes occurring at depth.

The episodic arrival of large gas bubbles in the lake causes spectacular Strombolian eruptions (Fig. 5). Although detailed studies have been made of these events using other techniques (Gerst et al., 2013; Jones et al., 2008), little has yet been learned of how they influence the pulsatory behaviour of the lake. Previous thermal camera systems on Erebus did not record the majority of the bubble events since their capture rate was too slow. However, the new system will allow explosive episodes to be recorded. Explosions can easily be identified in the data either as "shot noise" in the calculated surface motion or, for large events, as a sharp (factor of $\sim 5$ ) increase in the compressed size of the image files.

\section{Conclusions}

We have presented in detail the characteristics of a new thermal camera system, which was installed on Erebus volcano in November 2012 designed for continuous, year-round 
monitoring of the lava lake. In general, both hardware and software performed as expected from the outset and the continuity of the data set captured during the first year of deployment already exceeds any previously recorded. The failure of the wind generator and battery bank at the power generation site meant that the system did not run through the Antarctic winter in 2013, but it is hoped that this can be improved upon in future years. Images from the camera have been made publicly available at http://www.usap-data.org/ entry/NSF-ANT11-42083/ and this archive will be expanded each year as new data are retrieved from the camera.

The new thermal camera system has been something of a test project for the MEVO team into the feasibility of yearround monitoring from the crater rim of Erebus. Although there are clearly many challenges that must still be overcome before year-round data collection is a reality, we are now a few steps closer, and more "winter-over" instruments are already in the pipeline.

Acknowledgements. Field support for this project was provided by the NSF under award ANT1142083. Additional funding was received from European Research Council grant "DEMONS" (202844) under the European FP7, and the UK National Centre for Earth Observation "Dynamic Earth and Geohazards" theme (NERC NE/F001487/1: http://comet.nerc.ac.uk/). N. Peters wishes to thank B. McIntosh for his help with testing early versions of the system, Wookey for sharing his extensive knowledge of embedded Linux and E. Pacaud for his swift help in porting the Aravis library to ARM.

Edited by: L. Eppelbaum

\section{References}

Aster, R., McIntosh, W., Kyle, P., Esser, R., Bartel, B. A., Dunbar, N., Johns, B., Johnson, J. B., Karstens, R., Kurnik, C., McGowan, M., McNamara, S., Meertens, C., Pauley, B., Richmond, M., and Ruiz, M.: Real-time data received from Mount Erebus volcano, Antarctica, Eos, 85, 97-104, doi:10.1029/2004EO100001, 2004.

Bauguitte, S. J.-B., Brough, N., Frey, M. M., Jones, A. E., Maxfield, D. J., Roscoe, H. K., Rose, M. C., and Wolff, E. W.: A network of autonomous surface ozone monitors in Antarctica: technical description and first results, Atmos. Meas. Tech., 4, 645658, doi:10.5194/amt-4-645-2011, 2011.

Calkins, J., Oppenheimer, C., and Kyle, P.: Groundbased thermal imaging of lava lakes at Erebus volcano, Antarctica, J. Volcanol. Geoth. Res., 177, 695-704, doi:10.1016/j.jvolgeores.2008.02.002, 2008.
Dibble, R., Kyle, P., and Rowe, C.: Video and seismic observations of Strombolian eruptions at Erebus volcano, Antarctica, J. Volcanol. Geoth. Res., 177, 619-634, doi:10.1016/j.jvolgeores.2008.07.020, 2008.

Gerst, A., Hort, M., Aster, R. C., Johnson, J. B., and Kyle, P. R.: The first second of volcanic eruptions from the Erebus volcano lava lake, Antarctica - energies, pressures, seismology, and infrasound, J. Geophys. Res.-Sol. Earth, 118, 3318-3340, doi:10.1002/jgrb.50234, 2013.

Giggenbach, W. F., Kyle, P. R., and Lyon, G. L.: Present volcanic activity on Mount Erebus, Ross Island, Antarctica, Geology, 1, 135-136, doi:10.1130/00917613(1973)1<135:PVAOME>2.0.CO;2, 1973.

Huppert, H. E. and Hallworth, M. A.: Bi-directional flows in constrained systems, J. Fluid Mech., 578, 95-112, doi:10.1017/S0022112007004661, 2007.

Jones, K. R., Johnson, J. B., Aster, R., Kyle, P. R., and McIntosh, W.: Infrasonic tracking of large bubble bursts and ash venting at Erebus volcano, Antarctica, J. Volcanol. Geoth. Res., 177, 661-672, doi:10.1016/j.jvolgeores.2008.02.001, 2008.

Lawrence, J. S., Ashley, M. C. B., and Storey, J. W. V.: A remote, autonomous laboratory for Antarctica with hybrid power generation, J. Electronic Electrical Eng. Aust., 2, 1-12, 2004.

Magarey, J. and Kingsbury, N.: Motion estimation using a complexvalued wavelet transform, IEEE T. Signal Proces., 46, 10691084, doi:10.1109/78.668557, 1998.

Molina, I., Burgisser, A., and Oppenheimer, C.: Numerical simulations of convection in crystal-bearing magmas: a case study of the magmatic system at Erebus, Antarctica, J. Geophys. Res., 117, B07209, doi:10.1029/2011JB008760, 2012.

Oppenheimer, C., McGonigle, A. J. S., Allard, P., Wooster, M. J., and Tsanev, V.: Sulfur, heat, and magma budget of Erta 'Ale lava lake, Ethiopia, Geology, 32, 509-512, doi:10.1130/G20281.1, 2004.

Oppenheimer, C., Lomakina, A. S., Kyle, P. R., Kingsbury, N. G., and Boichu, M.: Pulsatory magma supply to a phonolite lava lake, Earth Planet. Sc. Lett., 284, 392-398, doi:10.1016/j.eps1.2009.04.043, 2009.

Orr, T. R. and Rea, J. C.: Time-lapse camera observations of gas piston activity at Pu'u 'Ō' ō, Kìlauea volcano, Hawai'i, B. Volcanol., 74, 2353-2362, doi:10.1007/s00445-012-0667-0, 2012.

Pacaud, E.: Aravis - GNOME Live!, available at: http://live.gnome. org/Aravis, last access: 22 May 2011.

Sawyer, G. M. and Burton, M. R.: Effects of a volcanic plume on thermal imaging data, Geophys. Res. Lett., 33, L14311, doi:10.1029/2005GL025320, 2006.

Spampinato, L., Calvari, S., Oppenheimer, C., and Boschi, E.: Volcano surveillance using infrared cameras, Earth-Sci. Rev., 106, 63-91, doi:10.1016/j.earscirev.2011.01.003, 2011.

Witham, F., Woods, A. W., and Gladstone, C.: An analogue experimental model of depth fluctuations in lava lakes, B. Volcanol., 69, 51-56, doi:10.1007/s00445-006-0055-8, 2006. 